\title{
Phenotypic-Genotypic Characterization and Enzyme Activity of Bacillus Spp. Isolated from Saricay Stream
}

\author{
Nurcihan Hacioğlu Doğru \\ Department of Biology, Faculty of Arts and Sciences, \\ Çanakkale Onsekiz Mart University, PO Box 17100, Turkey, \\ Tel: 90-286-218-0018 E-mail: nurcihan.n@gmail.com
}

\begin{abstract}
Derya Doğanay (Corresponding author)
Department of Oral and Dental Health, Vocational School of Health Services,

Biruni University, PO Box 34080, Turkey
\end{abstract}

Tel: 90-444-8276 E-mail: ddoganay@biruni.edu.tr

Received: October 16, 2017 Accepted: November 25, 2017 Published: November 30, 2017

Doi: 10.5296/jab.v6i1.12003ＵRL: http://doi.org/10.5296/jab.v6i1.12003

\begin{abstract}
63 bacteria consist of Bacillus genus were isolated from water samples of Saricay stream (Canakkale). Isolates were characterized for phenotypic properties, antimicrobial and heavy metal resistance and evaluated for some extracellular enzyme activities. 13 bacterial colonies were selected according to their different phenotypic-genotypic characteristics and described by $16 \mathrm{~S}$ rDNA sequence analysis methods.

There was a high incidence of resistance to Cefoxitin $(\mathrm{CN} 30 \mu \mathrm{g} / \mathrm{mL})$, while all of isolates sensitive against to Kanamycin $(\mathrm{K} 30 \mu \mathrm{g} / \mathrm{mL})$ and Chloramphenicol $(\mathrm{C} 30 \mu \mathrm{g} / \mathrm{mL})$. The trends in heavy metal resistance: Chromium $(\mathrm{Cr})>$ (Zinc) $\mathrm{Zn}>$ Copper $(\mathrm{Cu})>$ Manganese $(\mathrm{Mn})$. Totally 55 protease, 49 amylase, 30 cellulase and 59 lipase activities were determined from all of isolated Bacillus sp. None of the isolates showed the esterase activity. As a result of molecular identification six isolates were showed the highest similarity to different strain of Bacillus amyloliquefaciens and others similar to Bacillus sp. B22(2008), B. subtilis SD-45, Sporolactobacillus dextrus, Bacillus sp. IHBB3375, B. cereus RJ1, Uncultured organism clone ELU0176-T465-S-NIPCRAMgANa_000604, Uncultured organism clone ELU0110-T273-S-NI_000292.
\end{abstract}

This study is first of its kind to explore the bacterial enzyme resource from Saricay stream. This work forms the foundation for future research to enzyme characterization and assessed 
using in industrial fields explores the biotechnological potential of these Bacillus sp. isolates.

Keywords: Bacillus sp., Enzyme activity, 16s rDNA sequencing, Saricay stream

\section{Introduction}

In modern times, the products of biological origin, particularly enzymes, are attracting the attention of researchers. Their role in several biological and commercial processes has been duly emphasized. To meet the current increased demand, studies on the cost-effective production of industrially important enzymes have become necessary. Therefore, selection of the right organism plays a key role in high yield of desirable enzymes (Singh et al., 2010).

The potential contribution of marine and fresh water organisms to the discovery of new bioactive molecules is increasingly challenging (Parida et al., 2010). Bacterial organisms present in the fresh waters represent a potential source for enzyme production. Bacillus species have been major workhorse industrial microorganisms with roles in applied microbiology, including their high growth rates leading to short fermentation cycle times, their capacity to secrete proteins into the extracellular medium, and the GRAS (generally regarded as safe) status with the Food and Drug Administration for species (Schallmey et al., 2004). These spore-forming bacteria represent also a major micro flora in many natural biotopes, where they play an important role in ecosystem development; they are able to transform many chemical compounds (Mohanty et al., 2011).

In this study, we performed an investigation for Bacillus strains isolated from Saricay stream that are biotechnologically interesting for industrial application, by isolating and identifying thirteen strains that produced have better extracellular enzymes activities, and identified them with $16 \mathrm{~S}$ rDNA method.

\section{Materials and Methods}

\subsection{Study Area}

The Saricay stream is located in the southwest region of Marmara, latitude $39^{\circ}, 40^{\prime}-40^{\circ} 45^{\prime} \mathrm{N}$ and longuitude $25^{\circ}, 37^{\prime}-27^{\circ} 45^{\prime} \mathrm{E}$, in Canakkale, Turkey. This water resource is used for agriculture as on irrigation water and drinking water for animals (Hacioglu \& Dulger, 2010).

\subsection{Isolation of Bacillus sp. and Phenotypic Characterization}

All water samples were collected from Saricay stream, kept at $4{ }^{\circ} \mathrm{C}$ and brought under cold chain to laboratory. For isolation of Bacillus sp. and to destroy all the vegetative microbial cells water samples were incubated for $10 \mathrm{~min}$ at $80{ }^{\circ} \mathrm{C}$. Isolation and purification were done by dilution series method on nutrient agar (Oxoid) plates at $37^{\circ} \mathrm{C}$ for $24 \mathrm{~h}$. After incubation, the colonies with different morphological appearances selecting in order to phenotypic description and determine the enzyme activities (Topuz \& Kiran, 2007). Isolates were stained with Gram staining for purification control and determination of morphological features.

For taxonomic identification, the isolates were subjected to series of biochemical tests as described in Bergey's Manual of Systematic Bacteriology (Holt et al., 1994), which included catalase, oxidase, indole, Voges-Proskauer, citrate utilization, $\mathrm{H}_{2} \mathrm{~S}$ production, motility, acid production from mannitol, sucrose, glucose, growth at different $\mathrm{NaCl}$ concentrations (2-5-7-10\%), temperature (30-40-50 and $55^{\circ} \mathrm{C}$ ) and $\mathrm{pH}$ ranges (5 to 12$) .13$ bacterial colonies were selected according to their different morphological, physiological and biochemical characteristics. 


\subsection{Test of Antibiotic Sensitivity}

The inhibition of Bacillus strains by various antibiotics was tested by standard disc diffusion technique (Bauer et al., 1966). The following antibiotics were used; Kanamycin $(\mathrm{K} 30 \mu \mathrm{g} / \mathrm{mL})$, Oxytetracycline $(\mathrm{O} 30 \mu \mathrm{g} / \mathrm{mL})$, Cefmetazole $(\mathrm{CMZ30} \mu \mathrm{g} / \mathrm{mL})$, Furazolidone (FR50 $\mu \mathrm{g} / \mathrm{mL})$, Erythromycin $(\mathrm{E} 15 \mu \mathrm{g} / \mathrm{mL})$, Cefoxitin $(\mathrm{CN} 30 \mu \mathrm{g} / \mathrm{mL})$, Ampicillin (A10 $\mu \mathrm{g} / \mathrm{mL})$, Cefotaxime (CE30 $\mu \mathrm{g} / \mathrm{mL}$ ) and Chloramphenicol (C30 $\mu \mathrm{g} / \mathrm{mL})$ (CLSI, 2009).

\subsection{Determination of the Minimal Inhibitory Concentration (MIC) of Heavy Metals}

MIC for each bacterial isolate for four heavy metals was determined using Mueller - Hinton agar (Merck) containing $\mathrm{Zn}^{+2}, \mathrm{Cu}^{+2}, \mathrm{Cr}^{+3}$, and $\mathrm{Mn}^{+2}$ at concentrations ranging from 12.5 $\mu \mathrm{g} / \mathrm{mL}$ to $3200 \mu \mathrm{g} / \mathrm{mL}$. The metals were added as $\mathrm{ZnSO}_{4}, \mathrm{CuSO}_{4} \cdot 5 \mathrm{H}_{2} \mathrm{O}, \mathrm{K}_{2} \mathrm{Cr}_{2} \mathrm{O}_{7}$ and $\mathrm{MnCl}_{2} \cdot 2 \mathrm{H}_{2} \mathrm{O}$ (Merck). The isolates were considered resistant if the MIC values exceeded that of the E. coli K-12 strain which was used as the control (Matyar et al., 2008).

\subsection{Determination of Enzyme Activity of Isolates}

All of the isolates were screened for their protease, amylase, lipase, cellulase and esterase activities qualitatively on agar plates. Protease, amylolytic, lipase, esterase, cellulose activity were tested on Skim Milk Agar, M9-starch Agar, Tributyrin Agar, Tween 80 Medium, CMC agar, incubated at $37^{\circ} \mathrm{C}$ for $48 \mathrm{~h}$, respectively (Topuz \& K1ran, 2007; Ertugrul et al., 2007; Prabhavathy et al., 2013).

\subsection{Genomic DNA Isolation, $16 \mathrm{~S}$ rDNA Gene Amplification and Sequencing}

Genomic DNA was extracted from the cultures growing on nutrient agar for $18 \mathrm{~h}$ at $37^{\circ} \mathrm{C}$ by using genomic DNA purification kit (Fermentas). The 16S rDNA gene was amplified by using the bacterial universal primers $27 \mathrm{~F}$ (5'-AGA GTT TGA TCC TGG CTC AG-3') -1492 R (5'-GGT TAC CTT GTT ACG ACT T-3') as described previously [13]. PCR was carried out by Thermocycler (Techne, TC-512) in $0.2 \mu \mathrm{L}$ reaction tubes with $50 \mu \mathrm{L}$ final mixture volume. Each reaction mixture contained $20 \mathrm{pmol} / \mu \mathrm{L}$ concentration of $\mathrm{F}$ and $\mathrm{R}$ primers, 2 $\mathrm{mM}$ dNTP mix (Fermentas), $10 \mu \mathrm{L}$ of $10 \times$ PCR buffer, $25 \mathrm{mM} \mathrm{MgCl} 2,5 \mathrm{U} / \mu \mathrm{L}$ Taq DNA polymerase (Fermentas) and $3 \mu \mathrm{L}$ of genomic DNA. Amplification was performed according to the following program: preheating for $5 \mathrm{~min}$ at $94{ }^{\circ} \mathrm{C} ; 30$ cycles of denaturation for $1 \mathrm{~min}$ at $94{ }^{\circ} \mathrm{C}$; annealing for $1 \mathrm{~min}$ at $54{ }^{\circ} \mathrm{C}$; extension for $1 \mathrm{~min}$ at $72{ }^{\circ} \mathrm{C}$ and a final terminal extension for $10 \mathrm{~min}$ at $72{ }^{\circ} \mathrm{C}$ (Deng et al., 2010). PCR products were examined by $1 \%$ agarose gel electrophoresis in $1 \times$ TAE buffer at $100 \mathrm{~V}$. $1 \mathrm{~kb}$ DNA ladder (Fermentas) was used as a molecular size standard.

\subsection{Phylogenetic Analysis}

The sequences of $16 \mathrm{~S}$ rDNA genes were compared with those from the GenBank database using the NCBI Blast program. Sequences were aligned using the ClustalW program. Phylogenetic analyses were performed using the maximum-likelihood and neighbour-joining methods with bootstrap values based on 1000 replications. The phylogenetic tree (Saitou \& Nei, 1987) was constructed with the MEGA package version 4 (Tamura et al., 2007). As the phylogenetic analyses derived from neighbour-joining method were in congruence with those obtained using the maximum-likelihood algorithm, only the data obtained from the neighbour-joining method is presented in the study. 


\section{Al Macrothink}

\section{Results}

In this study, sixty three bacteria were isolated from Saricay stream and some biochemical tests, $\mathrm{pH}, \mathrm{NaCl}$ and temperature tolerance tests were performed for the determination of phenotypic characteristics of all isolates. All the strains were found to be Gram, catalase, motility, and oxidase positive, $\mathrm{H}_{2} \mathrm{~S}$ negative spore-forming Bacillus sp. rods. The isolates revealed variation in citrate $(57 \%)$ and VP $(25 \%)$ tests, indole utilization $(9.5 \%)$ and acid production from mannitol (17\%), sucrose (52\%), and glucose (92\%). The physiological studies showed the growth of all of isolates at $30-55^{\circ} \mathrm{C}$. 36 isolates tolerate to $2-10 \%$ and 27 isolates tolerate to $2-7 \%$ $\mathrm{NaCl}$ concentration; 56 isolates growth at $\mathrm{pH} \mathrm{5-10,7}$ isolates growth at $\mathrm{pH} 8-11$. Optimum growth temperature of all isolated bacteria was determined at $37^{\circ} \mathrm{C}$. Moreover all isolates were divided into 13 different groups according to phenotypic characteristics.

\subsection{Antimicrobial resistance of isolated bacteria}

The highest rates of resistance were against $\mathrm{CN} 30$ was the most commonly reported antimicrobial agent (85.71\%), followed by CMZ30 (15.87\%), O30 (14.28\%). Also resistant to other antibiotics observed in varying proportions. But all isolates sensitivity only two antibiotics - C30 and K30 - except B61, B36, respectively. However B7, B24, B35, B38, B43, B60 isolates have sensitive against all studied antibiotics (Table 1).

Table 1. Antibiotic resistance profile of isolated bacteria

\begin{tabular}{|c|c|c|c|c|c|c|c|c|c|}
\hline \multirow[b]{2}{*}{ Code } & \multicolumn{9}{|c|}{ Antibiotics } \\
\hline & $\begin{array}{l}\text { CN30 } \\
(n=54)\end{array}$ & $\begin{array}{l}\text { O30 } \\
(n=9)\end{array}$ & $\begin{array}{l}\text { FR50 } \\
(n=6)\end{array}$ & $\begin{array}{l}\text { A10 } \\
(n=8)\end{array}$ & $\begin{array}{l}\text { CMZ30 } \\
(n=10)\end{array}$ & $\begin{array}{l}\text { E15 } \\
(n=7)\end{array}$ & $\begin{array}{l}\text { CE30 } \\
(n=5)\end{array}$ & $\begin{array}{l}\text { C30 } \\
(n=1)\end{array}$ & $\begin{array}{l}\mathrm{K30} \\
(\mathrm{n}=1)\end{array}$ \\
\hline B1 & + & + & - & - & - & - & - & - & - \\
\hline B2 & + & - & - & - & - & - & - & - & - \\
\hline B3 & + & - & - & - & - & - & - & - & - \\
\hline B4 & + & - & - & - & - & - & - & - & - \\
\hline B5 & + & - & - & - & - & - & - & - & - \\
\hline B6 & + & + & - & - & - & - & - & - & - \\
\hline B7 & - & - & - & - & - & - & - & - & - \\
\hline B8 & + & - & + & - & - & + & - & - & - \\
\hline B9 & + & - & - & - & - & - & - & - & - \\
\hline B10 & + & - & - & - & - & - & - & - & - \\
\hline B11 & + & + & - & - & - & - & - & - & - \\
\hline B12 & + & - & - & - & - & - & - & - & - \\
\hline B13 & + & - & - & - & - & - & - & - & - \\
\hline B14 & + & - & - & - & - & - & - & - & - \\
\hline B15 & + & - & - & - & + & + & - & - & - \\
\hline B16 & + & - & - & - & - & - & - & - & - \\
\hline B17 & + & + & + & - & - & + & - & - & - \\
\hline B18 & + & + & - & - & - & - & - & - & - \\
\hline B19 & + & - & - & - & - & - & - & - & - \\
\hline B20 & + & - & - & - & - & - & + & - & - \\
\hline
\end{tabular}




\begin{tabular}{llll}
\hline $\mathbf{B} 21$ & + & - & - \\
B22 & + & - & - \\
B23 & + & - & \\
B24 & - & - & -
\end{tabular}

Table 1 (continued)

\begin{tabular}{|c|c|c|c|c|c|c|c|c|c|}
\hline \multirow[b]{2}{*}{ Code } & \multicolumn{9}{|c|}{ Antibiotics } \\
\hline & $\begin{array}{l}\text { CN30 } \\
(n=54)\end{array}$ & $\begin{array}{l}\text { O30 } \\
(n=9)\end{array}$ & $\begin{array}{l}\text { FR50 } \\
(n=6)\end{array}$ & $\begin{array}{l}\text { A10 } \\
(n=8)\end{array}$ & $\begin{array}{l}\text { CMZ30 } \\
(n=10)\end{array}$ & $\begin{array}{l}\text { E15 } \\
(n=7)\end{array}$ & $\begin{array}{l}\text { CE30 } \\
(n=5)\end{array}$ & $\begin{array}{l}\text { C30 } \\
(n=1)\end{array}$ & $\begin{array}{l}K 30 \\
(n=1)\end{array}$ \\
\hline B25 & + & - & - & - & - & - & - & - & - \\
\hline B26 & + & + & - & - & - & - & - & - & - \\
\hline B27 & + & + & - & - & - & - & - & - & - \\
\hline B28 & + & - & - & - & - & - & - & - & - \\
\hline B29 & + & - & - & - & - & + & - & - & - \\
\hline B30 & + & - & + & - & - & - & - & - & - \\
\hline B31 & + & + & - & - & - & - & - & - & - \\
\hline B32 & + & - & - & - & - & - & - & - & - \\
\hline B33 & + & - & - & - & - & - & - & - & - \\
\hline B34 & + & - & - & - & - & + & - & - & - \\
\hline B35 & - & - & - & - & - & - & - & - & - \\
\hline B36 & + & - & - & + & - & - & - & - & + \\
\hline B37 & + & - & - & - & - & - & - & - & - \\
\hline B38 & - & - & - & - & - & - & - & - & - \\
\hline B39 & + & + & - & - & - & - & - & - & - \\
\hline B40 & + & - & - & - & - & - & - & - & - \\
\hline B41 & + & - & - & - & + & - & - & - & - \\
\hline B42 & + & - & - & - & - & - & - & - & - \\
\hline B43 & - & - & - & - & - & - & - & - & - \\
\hline B44 & + & - & - & - & - & - & - & - & - \\
\hline B45 & + & - & - & - & - & - & - & - & - \\
\hline B46 & + & - & - & - & - & - & - & - & - \\
\hline B47 & + & - & - & - & - & - & - & - & - \\
\hline B48 & + & - & - & - & - & - & - & - & - \\
\hline B49 & + & - & - & - & - & - & - & - & - \\
\hline B50 & + & - & - & - & - & - & - & - & - \\
\hline B51 & + & - & - & + & + & - & - & - & - \\
\hline B52 & + & - & + & + & + & - & - & - & - \\
\hline B53 & + & - & - & - & - & - & - & - & - \\
\hline B54 & + & - & + & + & + & - & + & - & - \\
\hline B55 & + & - & - & - & + & - & - & - & - \\
\hline \multicolumn{10}{|c|}{ Table 1 (continued) } \\
\hline \multirow[b]{2}{*}{ Code } & \multicolumn{9}{|c|}{ Antibiotics } \\
\hline & $\begin{array}{l}\text { CN30 } \\
(n=54)\end{array}$ & $\begin{array}{l}\text { O30 } \\
(n=9)\end{array}$ & $\begin{array}{l}\text { FR50 } \\
(\mathrm{n}=6)\end{array}$ & $\begin{array}{l}\text { A10 } \\
(n=8)\end{array}$ & $\begin{array}{l}\text { CMZ30 } \\
(n=10)\end{array}$ & $\begin{array}{l}\text { E15 } \\
(n=7)\end{array}$ & $\begin{array}{l}\text { CE30 } \\
(n=5)\end{array}$ & $\begin{array}{l}\text { C30 } \\
(n=1)\end{array}$ & $\begin{array}{l}K 30 \\
(n=1)\end{array}$ \\
\hline
\end{tabular}




\begin{tabular}{llllllllll}
\hline B56 & + & - & - & + & + & - & + & - & - \\
B57 & + & - & - & + & + & - & - & - & - \\
B58 & + & - & - & + & + & - & + & - & - \\
B59 & + & - & - & - & - & - & - & - & - \\
B60 & - & - & - & - & - & - & - & - & - \\
B61 & - & - & - & - & - & + & - & + & - \\
B62 & + & - & - & - & - & - & - & - & - \\
B63 & + & - & - & - & - & - & - & - & - \\
\hline
\end{tabular}

\subsection{Heavy Metal Resistance}

In the present study, resistance to four heavy metals $\left(\mathrm{Mn}^{+2}, \mathrm{Cr}^{+3}, \mathrm{Zn}^{+2}\right.$ and $\left.\mathrm{Cu}^{+2}\right)$ was investigated for all isolates. The trends in heavy metal resistance are shown in Table 2; $\mathrm{Cr}$ $(100.00 \%)>\operatorname{Zn}(57.14 \%)>\mathrm{Cu}(55.55 \%)>\mathrm{Mn}(28.57 \%)$ (Table 2).

Table 2. Heavy metal tolerance of isolated bacteria

\begin{tabular}{|c|c|c|c|c|}
\hline \multirow{3}{*}{$\begin{array}{l}\text { Heavy } \\
\text { metal }\end{array}$} & $\begin{array}{l}M n>1600 \\
(n=18)\end{array}$ & \multirow{2}{*}{$\begin{array}{l}\mathrm{Cr}>100 \\
(n=63) \\
100 \%\end{array}$} & \multirow{2}{*}{$\begin{array}{l}\mathrm{Zn}>400 \\
(\mathrm{n}=36) \\
57.14 \%\end{array}$} & \multirow{2}{*}{$\begin{array}{l}\mathrm{Cu}>400 \\
(\mathrm{n}=35) \\
55.55 \%\end{array}$} \\
\hline & $28.57 \%$ & & & \\
\hline & B3,B4,B5,B6,B10,B11,B13, & B1-B63 & $\mathrm{B} 1, \mathrm{~B} 2, \mathrm{~B} 3, \mathrm{~B} 4, \mathrm{~B} 5, \mathrm{~B} 6$ & $\mathrm{~B} 1, \mathrm{~B} 2, \mathrm{~B} 3, \mathrm{~B} 4, \mathrm{~B} 5, \mathrm{~B} 6, \mathrm{~B} 7, \mathrm{~B} 10, \mathrm{~B} 11$, \\
\hline \multirow{9}{*}{$\begin{array}{l}\text { Code of } \\
\text { resistant } \\
\text { bacteria }\end{array}$} & $\mathrm{B} 14, \mathrm{~B} 15, \mathrm{~B} 18$ & isolated & B7,B9,B10,B11,B17, & B12, B13,B15,B16,B18,B19, \\
\hline & B24,B26,B2,7B28,B30,B33, & bacteria) & B18, B19,B20,B21, & $\mathrm{B} 20, \mathrm{~B} 21, \mathrm{~B} 27, \mathrm{~B} 28, \mathrm{~B} 29, \mathrm{~B} 31$, \\
\hline & B37, B56 & & B22, B26,B27,B28, & B33, \\
\hline & & & B30,B31, B33,B35, & B37, B38, B43,B44,B46,B47, \\
\hline & & & B36,B43,B44,B45, & B50, \\
\hline & & & B46,B48,B49, B51, & B53,B54,B56B59, B60, B63 \\
\hline & & & $\mathrm{B} 52, \mathrm{~B} 53, \mathrm{~B} 54, \mathrm{~B} 55$ & \\
\hline & & & B56,B57,B58, B60, & \\
\hline & & & B61 & \\
\hline
\end{tabular}

\subsection{Enzyme Capacity of Isolated Bacteria}

Extracellular enzyme activities of isolated bacteria were given in Table 3. Isolates showed protease $(n=55 ; 87 \%)$, amylase $(n=49 ; 78 \%)$, cellulose $(n=30 ; 48 \%)$ and lipase $(n=59 ; 94 \%)$ activity. But esterase enzyme potential is determined to be insufficient.

\section{$3.416 S$ rDNA Sequencing and Analysis}

According to phenotypic characteristics, isolates were divided into thirteen groups and molecular identification of selected isolates from each group was carried out. Molecular data and comparative phylogenetic tree of isolates were given in Table 4 and Figure 1, respectively. 
Table 3. Extracellular enzyme activity of isolated bacteria

\begin{tabular}{|c|c|c|c|c|c|c|c|c|c|c|c|}
\hline \multirow{2}{*}{ Code } & \multicolumn{5}{|l|}{ Enzyme } & \multirow{2}{*}{ Code } & \multicolumn{5}{|l|}{ Enzyme } \\
\hline & Protease & Amylase & Cellulase & Esterase & Lipase & & Protease & Amylase & Cellulase & Esterase & Lipase \\
\hline B1 & + & + & - & - & + & B33 & + & + & + & - & + \\
\hline B2 & + & + & - & - & + & B34 & - & - & - & - & - \\
\hline B3 & + & + & - & - & + & B35 & + & + & - & - & + \\
\hline B4 & + & + & + & - & + & B36 & + & + & + & - & + \\
\hline B5 & + & + & - & - & + & B37 & + & + & - & - & + \\
\hline B6 & + & + & - & - & + & B38 & + & + & - & - & + \\
\hline B7 & + & + & + & - & + & B39 & + & + & - & - & + \\
\hline B8 & - & - & - & - & - & B40 & + & + & + & - & + \\
\hline B9 & - & + & - & - & + & B41 & + & - & - & - & + \\
\hline B10 & + & - & + & - & + & B42 & + & + & - & - & + \\
\hline B11 & + & + & + & - & + & B43 & + & + & - & - & + \\
\hline B12 & + & + & - & - & + & B44 & + & + & + & - & + \\
\hline B13 & + & + & + & - & + & B45 & + & + & + & - & + \\
\hline B14 & + & + & - & - & - & B46 & + & + & + & - & + \\
\hline B15 & + & - & + & - & + & B47 & + & + & + & - & + \\
\hline B16 & + & + & + & - & + & B48 & + & + & + & - & + \\
\hline B17 & + & + & - & - & + & B49 & + & + & + & - & + \\
\hline B18 & + & + & + & - & + & B50 & + & + & + & - & + \\
\hline B19 & + & - & - & - & + & B51 & + & - & + & - & + \\
\hline B20 & + & - & - & - & + & B52 & + & - & + & - & + \\
\hline B21 & + & + & - & - & + & B53 & + & + & + & - & + \\
\hline B22 & + & - & - & - & + & B54 & + & + & + & - & + \\
\hline B23 & + & - & - & - & + & B55 & + & - & + & - & + \\
\hline B24 & + & + & + & - & + & B56 & - & + & + & - & + \\
\hline B25 & + & - & + & - & + & B57 & + & + & + & - & + \\
\hline B26 & + & + & - & - & + & B58 & + & + & + & - & + \\
\hline B27 & + & + & - & - & + & B59 & + & + & - & - & + \\
\hline B28 & + & + & - & - & + & B60 & - & + & - & - & + \\
\hline B29 & + & + & - & - & + & B61 & - & - & - & - & - \\
\hline B30 & + & + & + & - & + & B62 & - & + & - & - & + \\
\hline B31 & + & + & - & - & + & B63 & - & + & - & - & + \\
\hline B32 & + & + & + & - & + & & & & & & \\
\hline
\end{tabular}

Table 4. The 16S rDNA gene sequence similarities of the 13 Bacillus isolates to their closest relative(s)

\begin{tabular}{|c|c|c|c|c|}
\hline $\begin{array}{l}\text { Bacterial } \\
\text { Code }\end{array}$ & $\begin{array}{l}\text { Base } \\
\text { Number }\end{array}$ & $\begin{array}{l}\text { Reference from } \\
\text { database } \\
\text { Their similar species }\end{array}$ & $\begin{array}{l}\text { NCBI } \\
\text { Name of Strain }\end{array}$ & $\begin{array}{l}\text { Similarity } \\
\text { Percentage } \\
(\%)\end{array}$ \\
\hline B1 & 1434 & Bacillus amyloliquefaci & subsp.plantarum UCMB5113 & 100 \\
\hline
\end{tabular}




\section{I Macrothink}

\begin{tabular}{|c|c|c|c|c|}
\hline B3 & 1511 & Bacillus sp. & $\mathrm{B} 22(2008)$ & 99 \\
\hline B4 & 1471 & Bacillus subtilis & $\mathrm{SD}-45$ & 99 \\
\hline B6 & 952 & Sporolactobacillus dextrus & EH21 & 94 \\
\hline B8 & 1472 & Bacillus amyloliquefaciens & Aec 23 & 99 \\
\hline B9 & 887 & Bacillus amyloliquefaciens & $\mathrm{Zy} 25$ & 99 \\
\hline B15 & 1469 & Bacillus amyloliquefaciens & WY13 & 99 \\
\hline B19 & 935 & Bacillus sp. & IHB B 3375 & 99 \\
\hline $\mathrm{B} 23$ & 1431 & Bacillus amyloliquefaciens & Aec 23 & 96 \\
\hline B41 & 1446 & Uncultured organism clone & ELU0176-T465-S-NIPCRAMgANa_000604 & 99 \\
\hline B46 & 1512 & Bacillus amyloliquefaciens & subsp.plantarum UCMB5113 & 97 \\
\hline B48 & 1456 & Uncultured organism clone & ELU0110-T273-S-NI_000292 & 99 \\
\hline B54 & 1447 & Bacillus cereus & RJ1 & 100 \\
\hline
\end{tabular}

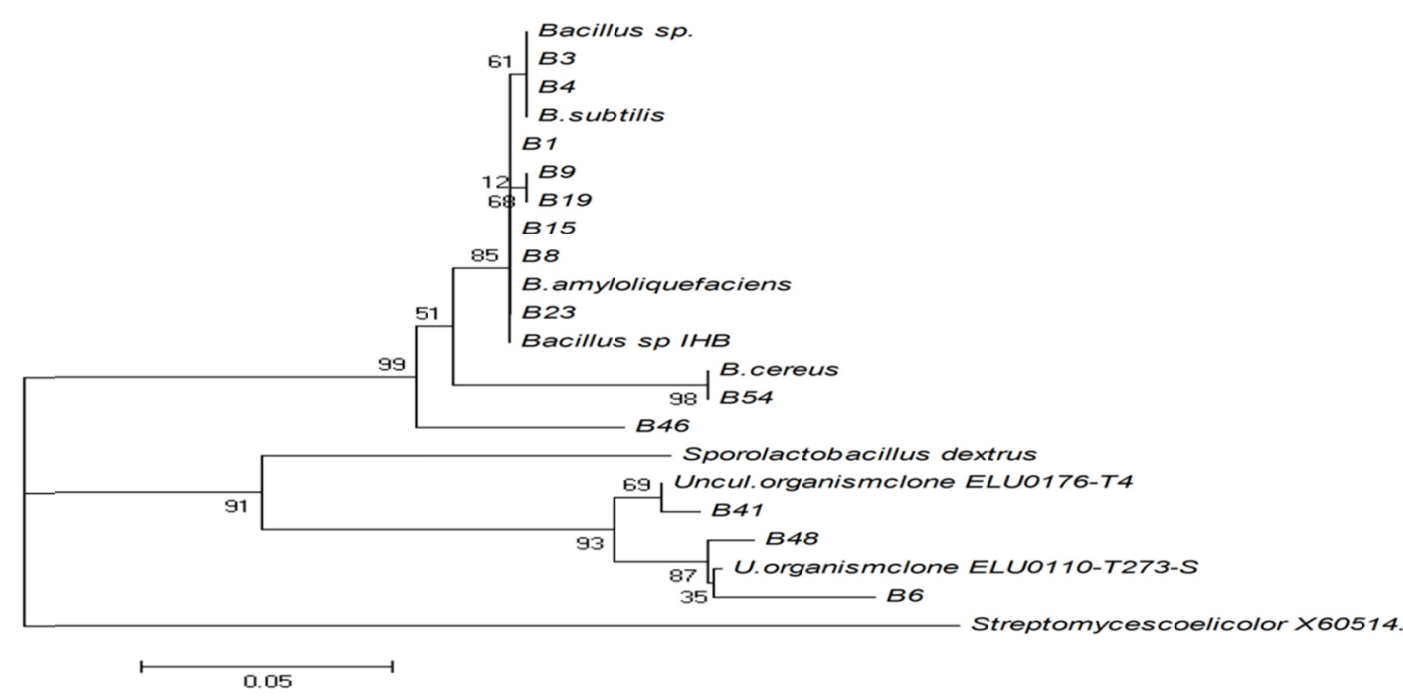

Figure 1. Phylogenetic tree showing the relative positions of identifiedl isolates by the neighbor-joining method of partial $16 \mathrm{~S}$ rDNA sequences

\section{Discussion}

This study on identification of Bacillus sp. isolated from Saricay stream (Canakkale) by biochemical tests, antimicrobial and heavy metal analysis and characterization of enzyme activity of isolated bacteria. Members of this genus are attractive industrial organisms in terms of important antibiotic and enzyme resources as well as their high growth rates and short fermentation cycles. Therefore, investigations concerning Bacillus sp. identification and biological products are very common (Singh et al., 2010; Schallmey et al., 2004; Ertugrul et al., 2007; Prabhavathy et al., 2013). On the contrary, research on freshwater Bacillus diversity and industrial potential are very limited (Mohanty et al., 2011). In addition to, although there were so many researches about microbial quality of Saricay stream (Hacioglu \& Dulger, 2010), this report will be first investigation on the bacterial diversity and their potential enzymatic activity. 
In our study obtained phenotypic traits showed that 7 out of 63 isolates were found to be alkaliphilic and others alkalotolerant Bacillus sp. All of the isolates also were determined as the mesophilic halo tolerant bacteria which is tolerant to different temperatures. In addition, all bacteria were divided into different 13 groups by the phenotypic properties of bacteria and 16S rDNA sequences of a bacterium from each group are analyzed for species identification. As a result of molecular identification six isolates were showed the highest similarity to different strain of B. amyloliquefaciens and others similar to Bacillus sp. B22(2008), B. subtilis SD-45, Sporolactobacillus dextrus, Bacillus sp. IHBB3375, B. cereus RJ1, Uncultured organism clone ELU0176-T465-S-NIPCRAMgANa_000604, Uncultured organism clone ELU0110-T273-S-NI_000292.

Because of the increasing enzyme technology and the variety usage of the products, industrial enzymes branch of biotechnology are gaining more and more importance. Therefore, quantitatively presence of industrially important protease, amylase, cellullase, lipase enzyme activity of isolated bacteria has been demonstrated. However, esterase enzyme potential of the isolates was found to be inadequate. It was determined that some of isolates also may be indicative more than one enzyme activity. Thus, researchers expressed that there are more than ten enzyme widely used in industry which is synthesized by Bacillus species and subspecies (Kumar \& Takagi, 1999). Our findings have a correlation of these reports.

When the antibiotic resistance of strains examined it has revealed that all strains have the high degree of resistance to different group antibiotics. There was a high incidence of resistance to $\mathrm{CN} 30$, while all of isolates sensitive against to $\mathrm{K} 30$ and $\mathrm{C} 30$ (except B36, B61, respectively). Antibiotics, for which the isolate is resistant, may be supplemented to fermentation medium during enzyme production process so as to check the contamination by other sensitive isolates (Singh et al., 2010). Antibiotic CN30 could be used for this purpose in enzyme production process from these isolated strains.

The isolated organisms also exhibited a high degree of tolerance to elevated concentrations $(\mu \mathrm{g} / \mathrm{mL})$ of $\mathrm{Cr}(100), \mathrm{Zn}(400)$ and $\mathrm{Cu}(400)$. In leather industries, this $\mathrm{Cr}$ tolerate isolates may be useful for dehairing process as the use of chromium is very common in the tanning process. Further, the protease producing organisms displaying heavy metal tolerance may be of potential use for the treatment of multi metal contaminated sludge generated during wastewater treatment (Singh et al., 2010). This case reveals that strains which has only resistance to antibiotics but also heavy metal resistant would be an important source of enzymes for many industrial processes.

It can be concluded that all isolated bacterial species are determined that they belong to the Bacillus genus with phenotypic and phylogenetic characteristics. The result is encouraging as bacteria with useful enzyme production potential are found in the fresh water of Saricay stream. The present study is a preliminary screening report of diversity of Bacillus species and their enzyme producing potential from Saricay stream. B. amyloliquefaciens can be expressed as a dominant species. Also in this study have not previously been cultured two Bacillus sp. species were isolated and cultured.

Isolation of Bacillus sp. and other industrial microorganisms from fresh water environment would also provide ample scope to assess their biotechnological potential (production of biotechnologically valuable compounds such as enzymes and antimicrobial substances). 
Attempt should be made for proper evaluation and exploration of these microbes for the biotechnological applications.

\section{References}

Bauer, A. W., Kirby, M. M., Sherris, J. C., \& Truck, M. (1966). Antibiotic susceptibility testing by a standardized single disk method. Am J Clin Pathol, 45, 493-6.

CLSI. (2009). Performance Standards for Antimicrobial Disk Susceptibility Tests; 88 Approved Standard-8 th ed. CLSI document M2-A8 (ISBN 1-56238-4856). CCLS, 940 West Valley Road, Suite 1400, Wayne, Pennsylvania 19087-1898 USA, 2009.

Deng, X., Long, S. H., He, D. F., Li, X., Wang, Y. F., Liu, J., \& Chen, X. B. (2010). Development and characterization of polymorphic microsatellite markers in Linum usitatissmum. J Plant Res, 123, 119-23. https://doi.org/10.1007/s10265-009-0271-3

Ertugrul, S., Donmez, G., \& Takac, S. (2007). Isolation of lipase producing Bacillus sp. from olive mill wastewater and improving its enzyme activity. J Hazard Mater, 149, 720-4. https://doi.org/10.1016/j.jhazmat.2007.04.034

Hacioglu, N., \& Dulger, (2010). B. Monthly variation of some physico-chemical and microbiological parameters in Saricay Stream (Canakkale, Turkey). Fresen Environ Bull, 19(5a), 986-90.

Holt, J. G., Krieg, N. R., \& Sneath, P. H. A. (1994). Staley JT. Bergey's Manual of Determinative Bacteriology. 9th ed. Williams and Wilkins Company, Balltimore, MD, USA; p. 255.

Kumar, C. G., \& Takagi, H. (1999). Microbial alkaline proteases: from a bio industrial viewpoint. Biotechnol Advances, 17, 561-94. https://doi.org/10.1016/S0734-9750(99)00027-0

Matyar, F., Kaya, A., \& Dincer, S. (2008). Antibacterial agents and heavy metal resistance in Gram-negative bacteria isolated from seawater, shrimp and sediment in Iskenderun Bay, Turkey. Sci Total Environ, 407, 279-85. https://doi.org/10.1016/j.scitotenv.2008.08.014

Mohanty, S., Choudhury, P. K., Dash, A., Samanta, M., \& Maiti, N. K. (2011). Genotypic and phenotypic diversity of Bacillus spp. isolated from freshwater ecosystems. J Aquac Res Dev, 2(2), 1-4.

Parida, S., Jena, R. C., Samal, K. C., \& Chand, P. K. (2012). Isolation and identification of pathogenic bacteria from Brackish Waters of Chilika Lagoon, Odisha, India for pharmaceutical use. Malays J Microbiol, 8(3), 197-202. https://doi.org/10.21161/mjm.04812

Prabhavathy, G. M., Rajasekara, P., \& Senthilkumar, B. (2013). Identification of Industrially important alkaline protease producing Bacillus subtilis by 16s rRNA sequence analysis and its applications. J Res Pharm Bio Sci, 4(1), 332-8.

Saitou, N., \& Nei, M. (1987). The neighbor-joining method: a new method for reconstructing phylogenetic tree. Mol Biol Evol, 4(4), 406-25.

Schallmey, M., Singh, A., \& Ward, O. P. (2004). Developments in the use of Bacillus species for industrial production. Can J Microbiol, 50, 1-17. https://doi.org/10.1139/w03-076

Singh, S. K., Tripathi, V. R., Jain, R. K., Vikram, S., \& Garg, S. K. (2010). An antibiotic, heavy metal resistant and halo tolerant Bacillus cereus SIU1 and its thermo alkaline protease. Microb Cell Fact, 9, 59-66. https://doi.org/10.1186/1475-2859-9-59 


\section{Macrothink}

Tamura, K., Dudley, J., Nei, M., \& Kumar, S. (2007). MEGA4: Molecular evolutionary genetics analysis (MEGA) software version 4.0. Mol Biol Evol, 24, 1596-9. https://doi.org/10.1093/molbev/msm092

Topuz, U., \& Kıran, O. E. (2007). Comlekcioglu U. Selülaz üreticisi Bacillus suslarının enzimatik özelliklerinin araştırılması. KSU J Sci Eng (In Turkish), 10(2), 13-6.

\section{Copyright Disclaimer}

Copyright reserved by the author(s).

This article is an open-access article distributed under the terms and conditions of the Creative Commons Attribution license (http://creativecommons.org/licenses/by/3.0/). 